\title{
The effect of silica-coating by sol-gel process on resin-zirconia bonding
}

\author{
Christie Ying Kei LUNG ${ }^{1}$, Edwin KUKK² and Jukka Pekka MATINLINNA ${ }^{1}$ \\ ${ }^{1}$ Dental Materials Science, Faculty of Dentistry, The University of Hong Kong, Hong Kong SAR, P.R. China \\ ${ }^{2}$ Department of Physics and Astronomy, Faculty of Mathematics and Natural Sciences, University of Turku, Finland \\ Corresponding author, Christie Ying Kei LUNG; E-mail: yklung@graduate.hku.hk
}

\begin{abstract}
The effect of silica-coating by sol-gel process on the bond strength of resin composite to zirconia was evaluated and compared against the sandblasting method. Four groups of zirconia samples were silica-coated by sol-gel process under varied reagent ratios of ethanol, water, ammonia and tetraethyl orthosilicate and for different deposition times. One control group of zirconia samples were treated with sandblasting. Within each of these five groups, one subgroup of samples was kept in dry storage while another subgroup was aged by thermocycling for 6,000 times. Besides shear bond testing, the surface topography and surface elemental composition of silica-coated zirconia samples were also examined using scanning electron microscopy and X-ray photoelectron spectroscopy. Comparison of silica coating methods revealed significant differences in bond strength among the Dry groups $(p<0.001)$ and Thermocycled groups $(p<0.001)$. Comparison of sol-gel deposition times also revealed significant differences in bond strength among the Dry groups $(p<0.01)$ and Thermocycled groups $(p<0.001)$. Highest bond strengths were obtained after 141-h deposition: Dry $(7.97 \pm 3.72 \mathrm{MPa})$; Thermocycled $(2.33 \pm 0.79 \mathrm{MPa})$. It was concluded that silica-coating of zirconia by sol-gel process resulted in weaker resin bonding than by sandblasting.
\end{abstract}

Keywords: Sol-gel processing, Resin composite, Zirconia, Bond strength, Silica-coating

\section{INTRODUCTION}

Yttria-stabilized tetragonal zirconia is widely used in dentistry as the material of choice for endodontic posts, implant abutments, single crowns, dental bridges, and dental subgingival implants ${ }^{1)}$. However, because of the inertness of zirconia, the bonding of dental resin composites to zirconia is inherently weak. To promote the adhesion of resin composites to zirconia in dental restorations ${ }^{2,3)}$, surface conditioning of zirconia is typically sandblasting with silica-coated alumina particles followed by application of silane coupling agent.

In dental laboratories, surface conditioning by sandblasting is carried out by blasting the substrate surface with silica-coated alumina particles forcibly propelled out of a nozzle under compressed air onto the surface ${ }^{4}$. Upon impact, the particles transfer their kinetic energy to the substrate surface. The energy absorbed by the substrate surface causes the latter to melt microscopically, allowing some silica-coated alumina particles to be incorporated within the substrate surface $^{5,6)}$. Finally, the silica-coated surface is primed by a silane coupling agent and the dental restoration cemented using a resin composite.

Other zirconia surface conditioning methods studied include chemical etching, laser irradiation, selective infiltration etching and chemical vapour deposition ${ }^{7-9)}$. All these methods were developed in order to enhance resin-zirconia bonding. In this study, another surface conditioning method of zirconia by sol-gel process is introduced. The applications for sol-gel-derived products are numerous, ranging from fabrication of integrated sensor systems, fiber optics, lasers, non-linear optical switching devices, and solid nanoparticles for drug delivery to thin-film coatings for dental implants and solar cells ${ }^{10-12)}$.

In dentistry, the sol-gel process has several advantages over the sandblasting method. Sandblasting potentially introduces surface defects or flaws. These surface irregularities generate compressive stresses on the surface layer, which adversely affect the long-term clinical performance of dental ceramics ${ }^{13}$. Another advantage is cost-related. Sol-gel processing requires less working space and less expensive equipment and chemical reagents as compared to the large and expensive sandblasting unit and silica-coated alumina sand particles used.

The purpose of this study was to evaluate the effects of different surface treatments for zirconia, sol-gel processing versus sandblasting, on the bond strength of resin composites bonded to zirconia. The null hypothesis was that different surface treatments and aging by thermocycling would have no influence on the shear bond strength of resin composites to zirconia. After sol-gel processing and sandblasting, surface elemental composition and surface topography of zirconia samples were also examined using X-ray photoelectron spectroscopy (XPS) and scanning electron microscopy (SEM) respectively.

\section{MATERIALS AND METHODS}

The materials used in this study are listed in Table 1. To simulate clinical conditions, materials used in clinical dentistry were employed in this study. 
Table 1 Materials used in this study

\begin{tabular}{|c|c|c|c|}
\hline Material & Manufacturer & Composition & Lot No. \\
\hline Zirconia (Lava $\left.{ }^{\mathrm{TM}}\right)$ & 3M ESPE & Yttria-stabilized zirconia & NA \\
\hline Sil Silane & 3M ESPE & $<3 \%$ 3-methacryloxypropyltrimethoxysilane & 259583 \\
\hline $\begin{array}{l}\text { Poly(methyl methacrylate) } \\
\text { resin }\end{array}$ & Ivoclar Vivadent AG & $\begin{array}{c}\text { Monomer: methyl methacrylate and } \\
\text { tetramethylene dimethacrylate } \\
\text { Polymer powder: poly(methyl methacrylate), } \\
\text { plasticizer, pigments, and catalyst }\end{array}$ & N69980 \\
\hline Tetraethyl orthosilicate & Sigma-Aldrich & $98 \%$ tetraethyl orthosilicate & 131903 \\
\hline Rocatec Sand Plus & 3M ESPE & $110 \mu \mathrm{m}$ silica-coated alumina & 369418 \\
\hline Deionized water & Millipore & $18.2 \mathrm{M} \Omega \mathrm{cm}$ resistivity & NA \\
\hline Ethanol & Riedel-de Haën & $99.8 \%$ ethanol & 03550 \\
\hline Ammonia & Sigma-Aldrich & $28-30 \%$ aqueous ammonia solution & 221228 \\
\hline RelyX Unicem Aplicap & 3M ESPE & $\begin{array}{l}\text { Powder: silanized fillers, initiator, pigments } \\
\text { Liquid: methacrylate monomers containing phosphoric } \\
\text { acid groups, initiator, stabilizers }\end{array}$ & 363991 \\
\hline
\end{tabular}

\section{Polishing of zirconia surfaces}

Zirconia blocks (Lava, 3M ESPE, Seefeld, Germany) of $16 \times 15 \times 3 \mathrm{~mm}^{3}$ dimensions were pressed into cylinder-shaped plastic molds filled with a poly(methyl methacrylate) resin (ProBase Cold, Ivoclar Vivadent AG, Schaan, Liechtenstein).

The surfaces of zirconia samples were polished with 400-grit silicon carbide papers under running deionized water. After ultrasonic cleaning in 70\% ethanol (Decon Laboratories Ltd., Hove, East Sussex, UK) for $10 \mathrm{~min}$, they were rinsed with 70\% ethanol and allowed to dry in air at room temperature.

\section{Silica-coating of zirconia by sol-gel process}

The sol-gel solvent mixture was prepared at room temperature using tetraethyl orthosilicate (TEOS; 98\%, Sigma-Aldrich, USA), absolute ethanol (99.8\%, Riedel-de Haën, Seelze, Germany), deionized water, and ammonia catalyst (28-30\%, Sigma-Aldrich).

Tetraethyl orthosilicate was added to a mixture of ethanol and deionized water, and then aqueous ammonia solution was added. Zirconia samples were immersed in the solvent mixture. The solution was slowly stirred. After the sol-gel deposition process, silica-coated zirconia samples were allowed to dry at room temperature, followed by rinsing with $70 \%$ ethanol and drying at room temperature. Four test groups of silica-coated zirconia samples by sol-gel process were thus prepared: Groups I to IV according to reagent ratios and deposition times (Table 2).

\section{Silica-coating of zirconia by sandblasting}

For control, a group of zirconia samples (Group V, Table 2) were silica-coated by sandblasting. Sandblasting was performed using Rocatec Sand Plus (110 $\mu \mathrm{m}$ of silica- coated alumina particles; 3M ESPE) at a constant pressure of $280 \mathrm{kPa}$ for $30 \mathrm{~s} / \mathrm{cm}^{2}$ and at a perpendicular distance of $10 \mathrm{~mm}$, according to standard procedure in dental laboratories ${ }^{14)}$.

Sandblasted zirconia samples were then ultrasonically cleaned in $70 \%$ ethanol for $10 \mathrm{~min}$. After rinsing with $70 \%$ ethanol, they were allowed to air-dry at room temperature.

Bonding of resin composite to silica-coated and silanized zirconia surfaces

Using a new fine brush for each application, one primer coating of Sil Silane (3M ESPE) solution was applied on silica-coated zirconia surfaces. 3M ESPE Sil Silane was a pre-hydrolyzed silane product consisting of 3-methacry loxypropyltrimethoxysilane ${ }^{15)}$. The coating was allowed to dry and react for $5 \mathrm{~min}$.

RelyX Unicem Aplicap resin composite (3M ESPE) was activated and mixed according to manufacturer's instruction. The resin composite was filled into a polyethylene mold of $3.7 \mathrm{~mm}$ diameter and $4.0 \mathrm{~mm}$ height, which was placed on the silica-coated and primed zirconia surface. After resin composite was light-cured for $40 \mathrm{~s}$ (Lunar Curing Light, Benlioglu Dental Inc., Ankara, Turkey), the mold was removed by being lifted up carefully.

Figure 1 illustrates the entire process of preparing the silica-coated (by sol-gel process or sandblasting) and silanized zirconia samples for shear bond strength measurement. Ten test groups of bonded samples were thus prepared: Groups I-V Dry and Groups I-V Thermocycled. Each test group consisted of 15 resin composite stubs for shear bond strength measurement. For the Dry samples of Groups I-V, they were kept in a desiccator at room temperature for $24 \mathrm{~h}$ prior to shear 
Table 2 Test groups I-V according to silica-coating method (sol-gel process or sandblasting) and according to reagent ratios and deposition times for sol-gel process

\begin{tabular}{|c|c|c|c|}
\hline Group & Surface treatment conditions & $\begin{array}{l}\text { TEOS:Ethanol: } \\
\text { Ammonia: } \\
\mathrm{H}_{2} \mathrm{O} \text { ratio }(\mathrm{mL})\end{array}$ & $\begin{array}{c}\text { Deposition } \\
\text { time }(\mathrm{h})\end{array}$ \\
\hline I & Polishing, rinsing (70\% ethanol), sol-gel process, rinsing (70\% ethanol) & 10:100:1:20 & 24 \\
\hline II & Polishing, rinsing (70\% ethanol), sol-gel process, rinsing (70\% ethanol) & $20: 100: 1: 20$ & 48 \\
\hline III & Polishing, rinsing (70\% ethanol), sol-gel process, rinsing ( $70 \%$ ethanol) & $5: 100: 2: 35$ & 72 \\
\hline IV & Polishing, rinsing (70\% ethanol), sol-gel process, rinsing (70\% ethanol) & $5: 100: 1: 15$ & 141 \\
\hline $\mathrm{V}$ & Polishing, rinsing (70\% ethanol), sandblasting, rinsing (70\% ethanol) & - & - \\
\hline
\end{tabular}

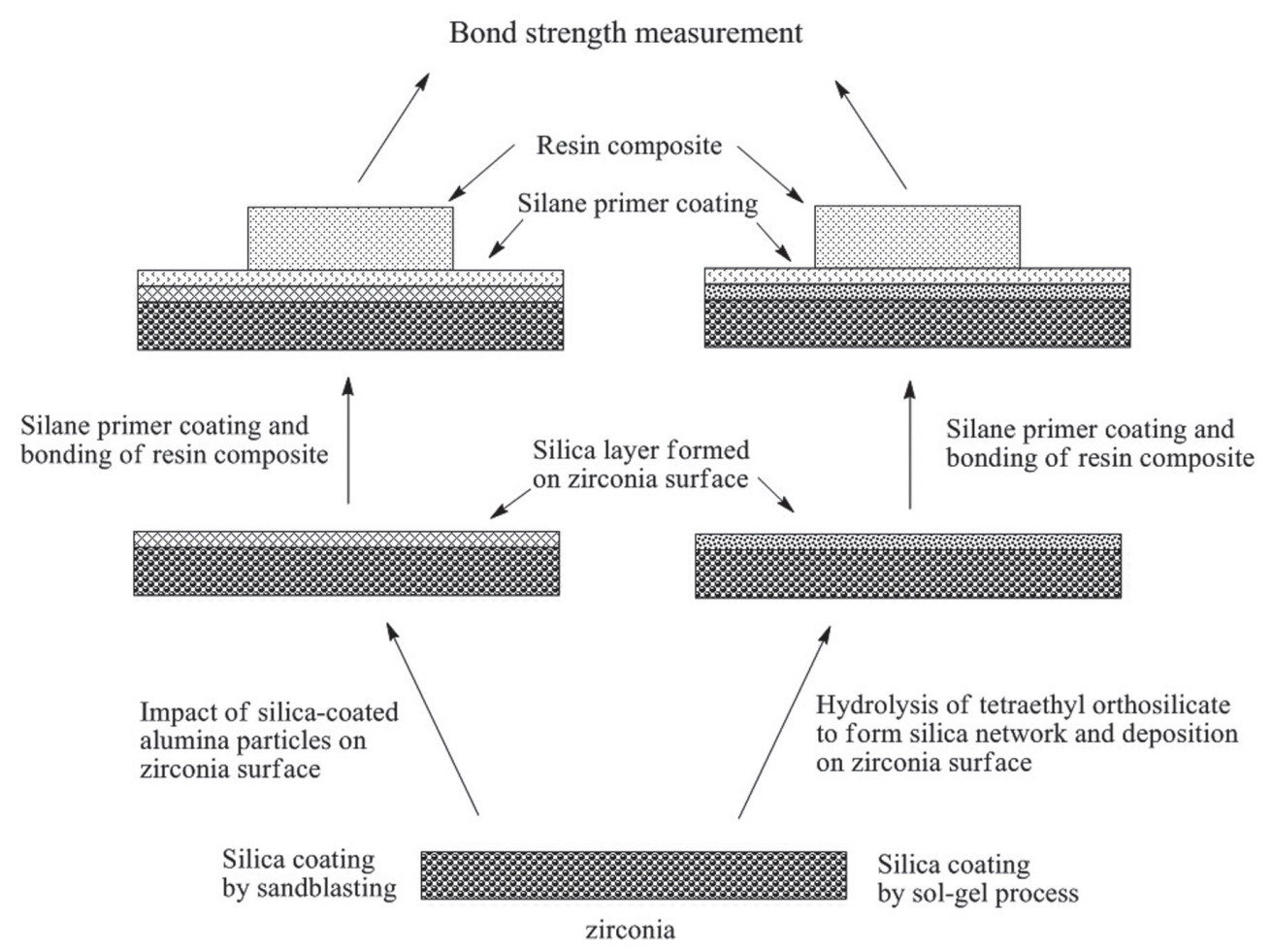

Fig. 1 Schematic illustration of bonding resin composites to silica-coated and silanized zirconia surfaces for shear bond strength testing using the sol-gel process or sandblasting method.

bond strength testing. For the thermocycled samples of Groups I-V, they were thermocycled 6,000 times using a custom-made thermocycling unit (Dental Materials Science Laboratory, University of Hong Kong, Hong Kong) between $5.0 \pm 0.5^{\circ} \mathrm{C}$ and $55.0 \pm 0.5^{\circ} \mathrm{C}$ with a dwell time of $20 \mathrm{~s}$ in each deionized water bath.

\section{Shear bond strength measurement}

Shear bond strengths (SBSs) of zirconia samples were measured using a universal testing machine (Model 1185, Instron, Norwood, MA, USA). A load of 1,000
$\mathrm{N}$ was applied at a crosshead speed of $1.0 \mathrm{~mm} / \mathrm{min}$ until fracture occurred. SBS was calculated using the maximum fracture load divided by the circular area of resin stub $^{15)}$.

After SBS testing, failure mode of each sample was assessed using a light microscope (NZ6, Leica, Wetzlar, Germany). Failure modes were classified as follows: 'adhesive failure' when less than $33 \%$ of resin composite remained on substrate surface; 'mixed failure 'when more than $33 \%$ but less than $66 \%$ of resin composite remained on substrate surface; and 'cohesive failure' 
when the remaining amount was more than $66 \%{ }^{14)}$.

\section{Statistical analysis of shear bond strengths}

Mean SBSs of resin composite to zirconia by sol-gel process using different deposition times and according to different surface conditioning methods were analyzed by one-way analysis of variance (ANOVA). Mean SBS was the dependent variable. Independent variables were namely: sol-gel deposition time, surface conditioning method (sol-gel process versus sandblasting), and storage condition (dry versus thermocycled). Inter-group comparisons of mean SBSs were performed using posthoc Tukey's test. Significance level for all comparisons was set at $p<0.05$.

Surface topography analysis

Surface topographies of zirconia samples after silicacoating by sol-gel process and sandblasting were analyzed by SEM (XL30CP, Philips Electron Optics, Eindhoven, The Netherlands) at $10 \mathrm{kV}$ operating voltage.

After SBS testing, representative zirconia samples were also analyzed by SEM (S-3400N, Hitachi, Tokyo, Japan) at $15 \mathrm{kV}$ operating voltage.

\section{XPS analysis}

The surface elemental compositions of five zirconia samples with surface treatments by sol-gel process and sandblasting (Table 2) were examined by XPS using a spectrometer (Perkin-Elmer PHI 5400, Waltham, MA, USA) with $\mathrm{MgK}_{\alpha}$ radiation $(\mathrm{h} v=1253.6 \mathrm{eV})$. Wide survey scans were performed to determine atomic concentrations. Chamber base pressure was about $8 \times 10^{-8} \mathrm{~Pa}$ and X-ray tube was operated at $200 \mathrm{~W}$. Peak composition and energy positions were determined using the least-squares curve-fitting technique with SPANCF macro package ${ }^{16)}$.

\section{RESULTS}

Shear bond strength

Table 3 shows the SBSs of resin composites to zirconia silica-coated by the sol-gel process or by sandblasting. Statistical analysis revealed that there were significant differences among different sol-gel deposition times within the Dry group $(p<0.01)$ and within the Thermocycled group $(p<0.001)$.

When comparison was made between the two surface conditioning methods of sol-gel processing versus sandblasting, significant differences were also found within the Dry group $(p<0.001)$ and within the Thermocycled group $(p<0.001)$.

On the effect of aging by thermocycling, the mean SBSs of all test groups were significantly decreased after thermocycling. Some zirconia samples of Groups I-IV even debonded during thermocycling.

\section{Failure mode}

According to the SEM micrographs in Fig. 2, all representative zirconia samples showed adhesive failure mode for both the sol-gel process and sandblasting treatment method.

Mode of failure assessment for all zirconia samples in all test groups was adhesive failure.

\section{Surface topography}

Figures 3(a)-(d) are the SEM micrographs that illustrate the surface topographies of silica-coated zirconia surfaces by sol-gel process. For deposition times of $24 \mathrm{~h}$ and 48 $\mathrm{h}$, there was non-uniform distribution of silica particles deposited in multiple layers on the zirconia surface (Figs. 3(a) and (b)). As the deposition time increased, there was more uniform distribution of the silica particles deposited on the zirconia surface (Figs. 3(c) and (d)).

Figure 3(e) shows that after sandblasting, silicacoated alumina particles deposited on the zirconia surface were finely distributed.

Figure 3(f) shows the surface topography of polished zirconia surface without any surface treatment by the sol-gel process or by sandblasting.

\section{Surface elemental composition}

Table 4 shows the elemental compositions of silica-

Table 3 Mean shear bond strengths of resin composite to zirconia by sol-gel processing at different deposition times and by sandblasting

\begin{tabular}{cccccc}
\hline \multirow{2}{*}{ Group } & Silica coating method & Deposition Time (h) & \multicolumn{2}{c}{ Mean SBS \pm SD (MPa) } & Debonded during \\
thermocycling (\%)
\end{tabular}

Different capital letters in the same row mean that groups are significantly different $(p<0.05)$.

Different lowercase letters in the same column mean that groups are significantly different $(p<0.05)$. 


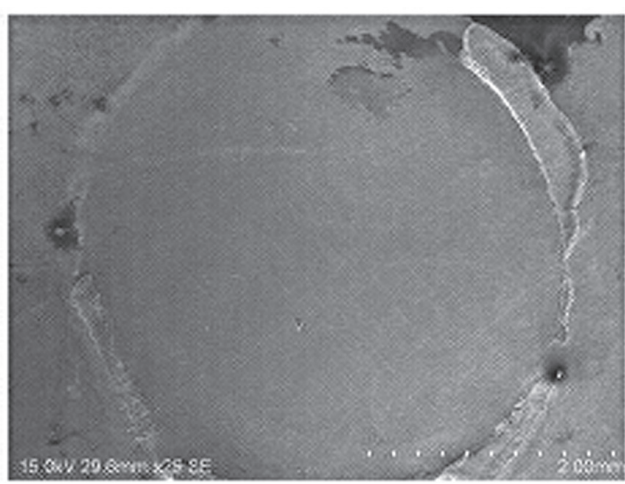

(a)

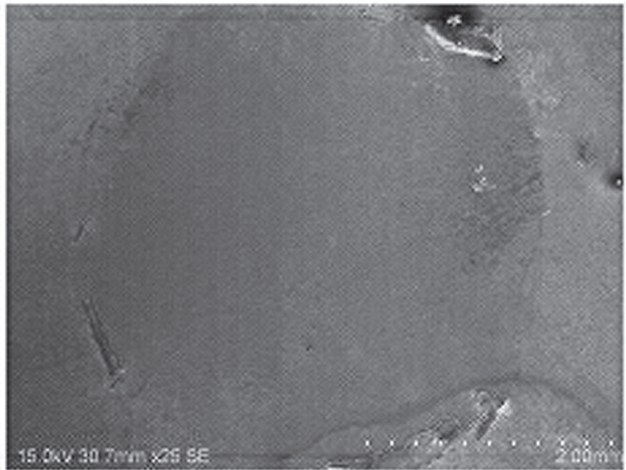

(c)

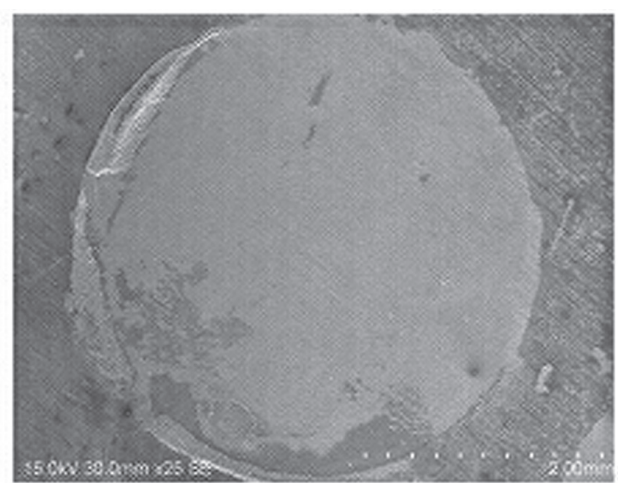

(b)

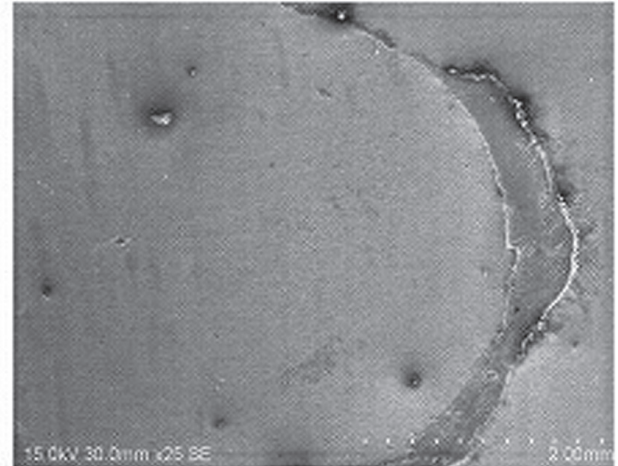

(d)

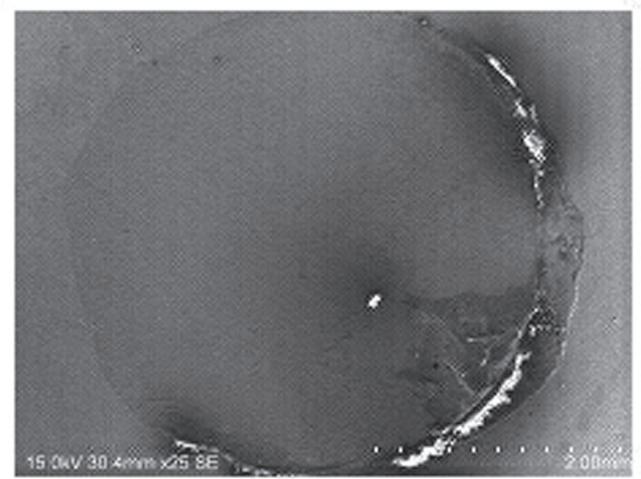

(e)

Fig. 2 SEM micrographs $(25 \times)$ which show resin composite remaining on Dry zirconia samples after SBS testing.
(a) 24-h deposition; (b) 48-h deposition;
; (c) 72-h deposition;
(d) 141-h deposition; and

(e) sandblasting.

coated zirconia surfaces, after sol-gel processing or sandblasting, as examined by XPS. For samples 1-4 after the sol-gel process, only elemental $\mathrm{C}, \mathrm{Si}$, and $\mathrm{O}$ were detected on the surface layer. For sample 5 after sandblasting, elemental $\mathrm{C}, \mathrm{O}, \mathrm{Zr}, \mathrm{Si}$, and $\mathrm{Al}$ were detected. $\mathrm{Si}$ and $\mathrm{Al}$ contents in Group V samples were due to the use of silica-coated alumina particles during sandblasting. Further comparison between the two silica-coating methods revealed that $\mathrm{Si}$ content in sample 1-4 was higher than in sample 5 .

\section{DISCUSSION}

In this study, the effect of silica-coating on zirconia by sol-gel process on the shear bond strength of resin composites to zirconia was evaluated. It was found that bond strength increased with deposition time for both dry and thermocycled samples. However, in routine clinical practice, it is quite impractical to implement such long sol-gel deposition times. 


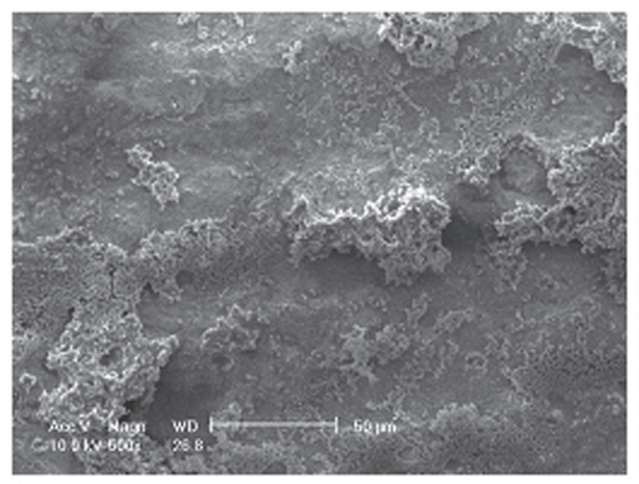

(a)

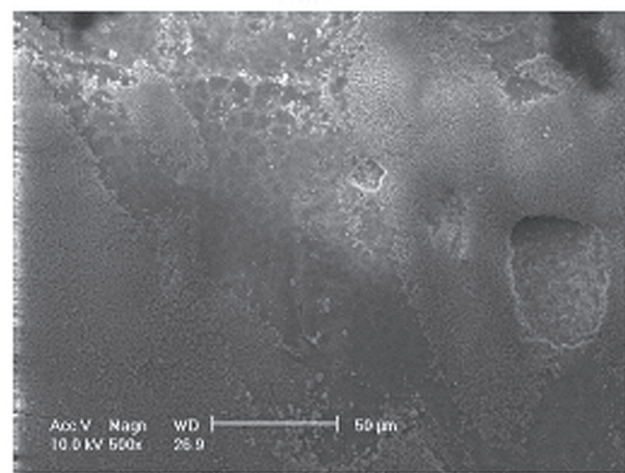

(c)

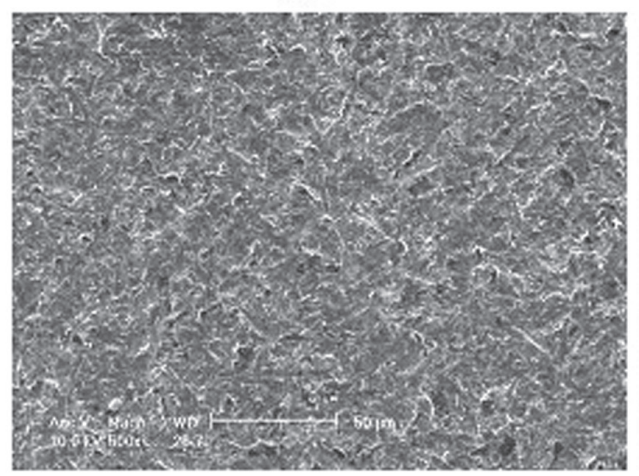

(e)

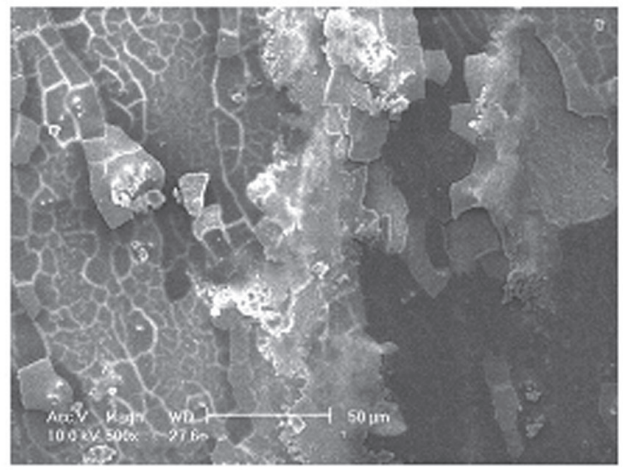

(b)

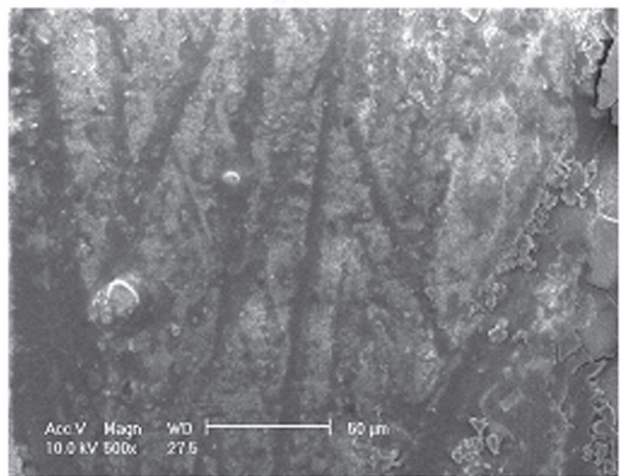

(d)

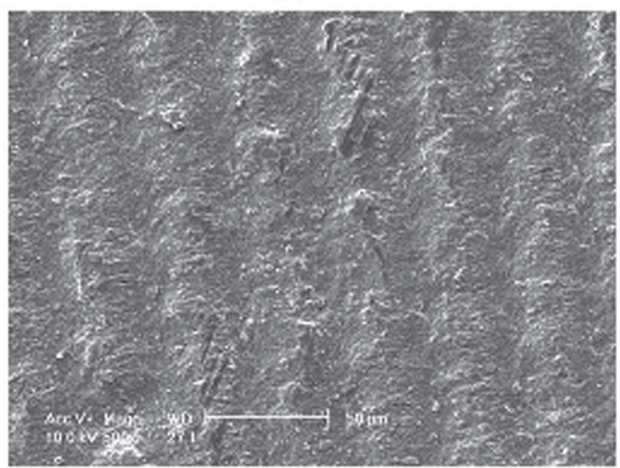

(f)

Fig. 3 SEM micrographs (500x) of: (a-d) Silica-coating of zirconia by sol-gel process after deposition of 24 h (a), 48 h (b), 72 h (c), 141 h (d); (e) Silica-coating by sandblasting; and (f) Polished zirconia surface without surface conditioning.

Table 4 XPS elemental compositions of zirconia surfaces after sol-gel processing and sandblasting

\begin{tabular}{|c|c|c|c|c|c|c|}
\hline \multirow{2}{*}{ Sample } & \multirow{2}{*}{ Silica coating method } & \multicolumn{5}{|c|}{ Atomic concentration (\%) } \\
\hline & & $\mathrm{Zr}_{3 \mathrm{~d}}$ & $\mathrm{O}_{1 \mathrm{~s}}$ & $\mathrm{Si}_{2 \mathrm{p}}$ & $\mathrm{C}_{1 \mathrm{~s}}$ & $\mathrm{Al}_{2 \mathrm{p}}$ \\
\hline 1 & Sol-gel process & & 54.0 & 26.9 & 19.1 & \\
\hline 2 & Sol-gel process & & 62.4 & 28.6 & 9.0 & \\
\hline 3 & Sol-gel process & & 38.6 & 20.4 & 41.0 & \\
\hline 4 & Sol-gel process & & 54.9 & 27.4 & 17.7 & \\
\hline 5 & Sandblasting & 7.2 & 44.0 & 9.9 & 31.0 & 7.9 \\
\hline
\end{tabular}




\section{Effect of aging by thermocycling}

Thermocycling is a common artificial aging method used in dental research to test bond durability, where the bonding interface is exposed to the combined effects of hydrolytic degradation and thermal stress. In the present study, all samples from Groups I-V showed significant decrease in shear bond strength after thermocycling. In particular, samples with $24 \mathrm{~h}$ and 48 $\mathrm{h}$ of sol-gel deposition showed near-zero bond strength after thermocycling.

Failure mode analysis indicated adhesive failure in both dry and thermocycled samples of Groups I-V. In other words, interfacial failure occurred between the resin composite and silica-coated layer on zirconia. Some samples from Groups I-IV even debonded during thermocycling. Therefore, it was apparent that resin-zirconia bonding was adversely affected by thermal fatigue and hydrolytic degradation during thermocycling.

\section{Effect of silica-coating by sandblasting}

For the sandblasting process, tribochemical coating process is the mechanism by which silica particles are deposited on the zirconia surface. Tribochemistry involves creating chemical bonds by applying mechanical energy. During sandblasting, chemical reactions are initiated by frictional energy and shearing forces between the lubricant (silica-coated alumina particles) and the substrate surface (zirconia) under boundary lubrication conditions ${ }^{17)}$.

In the present study, the two sources of tribochemistry were: (1) chemical reactions induced by mechanical impact; and (2) chemical reactions induced by frictional heat generated between the silica-coated alumina particles and zirconia surface ${ }^{18)}$. First, mechanical impact caused the interatomic bonds of surface atoms to stretch, hence reducing the activation barrier of interfacial reactions ${ }^{19)}$. Secondly, frictional heat caused the surface temperature to rise and reach the maximum temperature, called the 'flash temperature'. Rise to the maximum temperature also increased the rate of chemical reactions between the silica-coated alumina particles and zirconia surface ${ }^{20)}$.

\section{Effect of silica-coating by sol-gel process}

For the sol-gel process, the formation of a silica network involves two steps: hydrolysis and polycondensation of tetraethyl orthosilicate ${ }^{21)}$. Tetraethyl orthosilicate undergoes hydrolysis to form silanols. Two silanol molecules condense to form a siloxane linkage, which then undergoes polycondensation to form a silica network.

Silica particles chemically react with the zirconia surface, through the interaction of hydroxyl groups of silica particles with the surface hydroxyl groups of zirconia, to form a silica layer on the zirconia surface. Besides, hydrolyzed tetraethyl orthosilicate may also condense on the zirconia surface first and then build up to form a silica layer on it.

\section{Effect of silane coupling agents}

Silane coupling agents, such as 3M ESPE Sil Silane, are used to promote adhesion of resin composites to dental restorative materials ${ }^{9)}$. They contain two different functional groups: $>\mathrm{C}=\mathrm{C}<$ vinyl double bond and $-\mathrm{OR}$ group. The former can react with the functional groups of resin composites consisting of $>\mathrm{C}=\mathrm{C}<$ bonds. The latter forms $-\mathrm{OH}$ after hydrolysis, which then reacts chemically with the silica-coated zirconia surface.

In the present study, the statistically significant difference in SBS between the two silica coating methods was largely due to the mechanism of silica deposition on the zirconia surface. With sandblasting, a silica layer was formed on the zirconia surface after the surface was blasted with silica-coated alumina particles, followed by deposition of silica particles on the surface. For the sol-gel process, a silica layer was formed on the zirconia surface by the hydrolysis of tetraethyl orthosilicate to form silanols. Condensation of silanols then resulted in the formation and deposition of silica particles on the zirconia surface.

After silica-coating zirconia by both methods, a silane coupling agent was applied. The silane coupling agent formed silanols after hydrolysis, which then reacted with the surface hydroxyl groups of the silica layer on zirconia surface to form siloxane linkages (-O-Si-O-) after condensation. Finally, resin composite was bonded to the silica-coated and silanized zirconia surface by lightcuring. During polymerization, the $>\mathrm{C}=\mathrm{C}<$ bonds in the composite resin reacted with the $>\mathrm{C}=\mathrm{C}<$ bonds in the silane coupling agent to form $-\mathrm{C}-\mathrm{C}-$ single bonds.

Given the explanation above, it became clear that difference in SBS between the sandblasting treatment and sol-gel process stemmed from the difference in adhesion between the silica layer and the zirconia surface, since the bonding mechanism was the same between the resin composite and the silane primer coating in both methods.

\section{Future work}

Our aim was to develop a novel zirconia surface conditioning method to improve the adhesion of resin composites to zirconia. This method must be costeffective, energy-saving, and easy in implementation to both dentists and dental technicians. Further studies would be carried out to investigate the silica-coating of zirconia by sol-gel process with optimized modifications as well as by other surface conditioning methods.

\section{CONCLUSIONS}

Within the limitations of the current study, the following conclusions were drawn:

1. The null hypothesis was rejected. Two factors significantly affected the shear bond strength of resin composite to zirconia: silica coating method of zirconia (sol-gel process or sandblasting) and aging effect of thermocycling.

2. Shear bond strength increased with sol-gel deposition time, but it is impractical in clinical practice for long deposition times. 


\section{ACKNOWLEDGMENTS}

This study was financially supported by research grants from The University of Hong Kong. The authors wish to thank 3M ESPE for generously supplying the resin composite and silane coupling agent. Markku Heinonen, The University of Turku, is acknowledged for his assistance in XPS analysis.

\section{REFERENCES}

1) Nothdurft FP, Motter PJ, Pospiech PR. Effect of surface treatment on the initial bond strength of different luting cements to zirconium oxide ceramic. Clin Oral Investig 2009; 13: 229-235.

2) Thompson JY, Stoner BR, Piascik JR, Smith R. Adhesion/ cementation to zirconia and other non-silicate ceramics: where are we now? Dent Mater 2011; 27: 71-82.

3) Matinlinna JP, Lassila LV. Enhanced resin-composite bonding to zirconia framework after pretreatment with selected silane monomers. Dent Mater 2011; 27: 273-280.

4) Matinlinna JP, Vallittu PK. Bonding of resin composities to etchable ceramic surfaces - an insight review to the chemical aspects on surface conditioning. J Oral Rehabil 2007; 34: 622-630.

5) Heikkinen TT, Lassila LVJ, Matinlinna JP, Vallittu PK. Effect of operating air pressure on tribochemical silicacoating. Acta Odontol Scand 2007; 65: 241-248.

6) Gbureck U, Masten A, Probst J, Thull R. Tribochemical structuring and coating of implant metal surfaces with titanium oxide and hydroxyapatite layers. Mater Sci Eng 2003; 23: 461-465.

7) Lung CYK, Matinlinna JP, Kukk E, Hägert T. Surface modification of zirconia by various chemical treatments. Appl Surf Sci 2010; 257: 1228-1235.
8) Ural Ç, Külünk T, Külünk Ş, Kurt M. The effect of laser treatment on bonding between zirconia ceramic surface and resin cement. Acta Odontol Scand 2010; 68: 354-359.

9) Lung CYK, Matinlinna JP. Aspects of silane coupling agents and surface conditioning in dentistry: an overview. Dent Mater 2012; 28: 467-477.

10) Gvishi R. Fast sol-gel technology: from fabrication to applications. J Sol-Gel Sci Technol 2009; 50: 241-253.

11) Avnir D, Coradin T, Lev O, Livage J. Recent bio-applications of sol-gel materials. J Mater Chem 2006; 16: 1013-1030.

12) Guo CY, Tang ATH, Matinlinna JP. Insights into surface treatment methods of titanium dental implants. J Adhes Sci Technol 2012; 26: 189-205.

13) Zhang Y, Lawn BR, Rekow ED, Thompson VP. Effect of sandblasting on the long-term performance of dental ceramics. J Biomed Mater Res Part B: Appl Biomater 2004; 71B: 381-386

14) Lung CYK, Matinlinna JP. Resin bonding to silicatized zirconia with two isocyanatosilanes and a cross-linking silane. Part I: Experimental. Silicon 2010; 2: 153-161.

15) Matinlinna JP, Lassila LVJ, Vallittu PK. The effect of five silane coupling agents on the bond strength of a luting cement to a silica-coated titanium. Dent Mater 2007; 23: 1173-1180.

16) Kukk E. Spectral Analysis by Curve Fitting (SPANCF). Available from: http://www.physics.utu.fi/en/research/ material_science/Fitting.html, 2009.

17) LiSH, PalekarV. Quantum mechanochemistry understanding of tribochemical reactions. Tribol Mater Surf Interfaces 2009; 3: $132-142$.

18) Hsu SM, Zhang J, Yin Z. The nature and origin of tribochemistry. Tribol Lett 2002; 13: 131-139.

19) Muratov VA, Fischer TE. Tribochemical polishing. Ann Rev Mater Sci 2000; 30: 27-51.

20) Fischer TE. Tribochemistry. Ann Rev Mater Sci 1988; 18: 303-323.

21) Buckley AM, Greenblatt M. The sol-gel preparation of silica gels. J Chem Educ 1994; 71: 599-602. 